\title{
Size and Shape Control of Gold Nanodeposits in an Array of Silica Nanowells on a Gold Electrode
}

\author{
Amy E. Rue and Maryanne M. Collinson \\ Department of Chemistry, Virginia Commonwealth University, Richmond, VA 23284, USA \\ Correspondence should be addressed to Maryanne M. Collinson, mmcollinson@vcu.edu \\ Received 7 May 2011; Revised 26 October 2011; Accepted 27 October 2011 \\ Academic Editor: Tebello Nyokong
}

Copyright ( $) 2012$ A. E. Rue and M. M. Collinson. This is an open access article distributed under the Creative Commons Attribution License, which permits unrestricted use, distribution, and reproduction in any medium, provided the original work is properly cited.

Ordered arrays of hemispherical nanowells were formed in a sol-gel-derived silica film on a gold electrode using $500 \mathrm{~nm}$ diameter polystyrene latex spheres as templates. The conductive domain located at the bottom of each nanowell upon template removal was enlarged via electroless deposition from a gold plating solution. The structured electrodes thus formed were characterized using scanning electron microscopy and atomic force microscopy. Depending on the method used to make the films, the extent of the long-range packing and the size of the conductive domain changed. Electroless deposition in the nanowells produced (near) sphere-like nanostructures of gold, the size of which depended on the incubation time in the plating solution and the size of the conductive domain. Longer exposure times yielded nanostructures that filled the nanowell, whereas smaller exposure time yielded much smaller structures. Significantly larger, rougher deposits were formed in nanowells with large conductive domains. The electrochemical response observed at these electrodes was strongly dependent on the extent of long-range packing, the presence of defect sites in the film and their relative spacing, and the redox species in solution.

\section{Introduction}

Patterned or structured electrodes have been shown to have considerable importance in many areas of science and technology, particularly electroanalytical applications [1-3]. The development of strategies for the fabrication of such materials is needed as the desire to make smaller, more sensitive, and more selective devices becomes increasingly important. One means to create structured electrodes involves strategically combining sol-gel chemistry and templating together $[4,5]$. In such a process, for example, a suitable template is doped into a silica sol and cast on a surface such as an electrode. Upon subsequent removal of the template, voids remain in the microporous oxide network. Examples of various types of templates that have been used include molecules, surfactants, and latex spheres [3-7]. In prior work, we have shown how an array of silica nanowells can be formed on an electrode surface and how these structured electrodes can be used to electrochemically grow copper nanoparticles and conducting polymers from the bottom-up [8]. The success of this method depends on the extent at which, upon removal of the latex sphere, the underlying electrode surface is exposed, enabling it to be used as a microelectrode [9]. Providing there are no defects (pin holes) in the insulating silica surrounding the nanowells, the electrochemistry only takes place in the nanowell, thus restricting growth to this area [8]. The advantages of growing arrays of nanoparticles within a porous inorganic network using this approach are the following: (a) the interparticle spacing between nanoparticles can be controlled by the size of the template used to form the nanowell, (b) the size and shape of the resultant nanoparticles/nanostructures can be controlled by the size and shape of the nanowell, and (c) the silica support helps prevent aggregation of the nanoparticles as they begin to grow larger $[8,10,11]$. From an electroanalytical point of view, enlarging the conductive domains located at the bottom of the nanowell also provides a means to potentially increase the sensitivity of the microelectrode array [12]. The importance of nanoparticle arrays in electroanalytical chemistry has been well documented in the literature [12$15]$. 

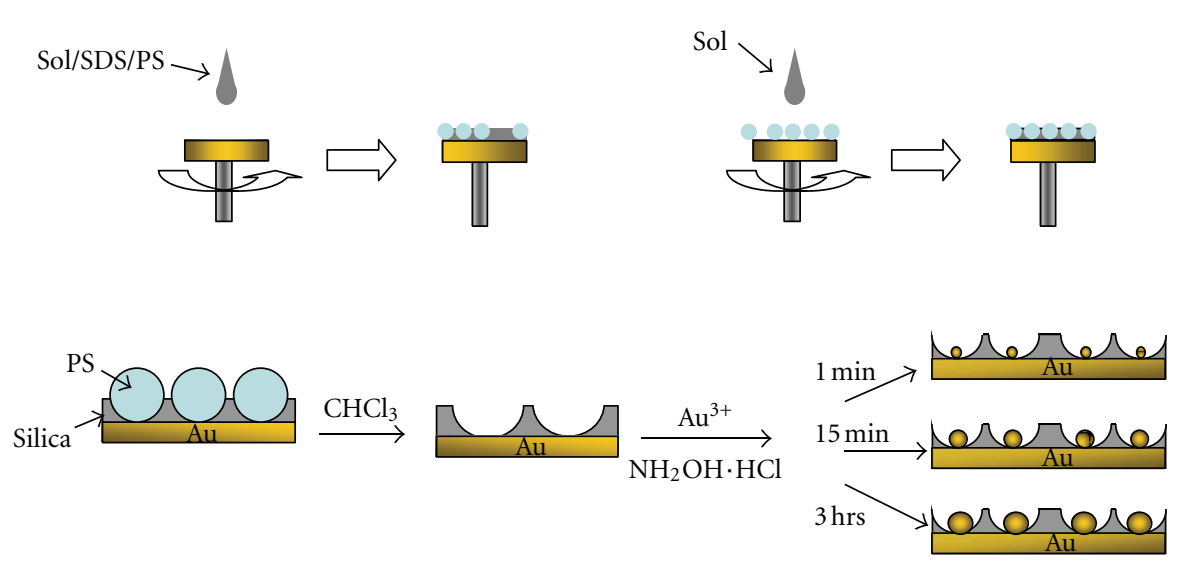

Figure 1: Simplified cartoon that shows the fabrication of silica nanowells on the gold electrode and the subsequent enlargement of the conductive domains exposed after template removal.

In this work, we describe an alternative approach for expanding the conductive domain at the bottom of the porous nanowells that is less sensitive to defect sites (pin holes) in the silica framework and more amenable to different metals. The procedure involves the formation of the silica nanowells on a gold electrode using polystyrene latex spheres as the sacrificial template followed by the electroless growth of the gold microelectrode that is exposed when the template is removed. The height of the gold nanoparticles can be easily adjusted by changing the incubation time in the electroless plating solution. In related work recently published, the silica nanowells were formed from the vapor deposition of dimethyldichlorosilane around an array of latex spheres formed on quartz. To form the gold nanoparticle array, the exposed surface (hydroxides) was immersed in a solution of tin ions, followed by silver ions, and then gold ions [11]. In another paper, shallow nanowells were formed on a silicon wafer via the vapor deposition of chlorosilanes around the latex sphere $[10,16,17]$. The hydroxylated surface located at the bottom of the nanowells was then functionalized with mercaptopropyltrimethoxysilane and subsequently used to bind gold nanoparticles [10]. In contrast to these papers, the current method is a one-step method that does not require multiple reaction steps or chemical modification. It utilizes the sol-gel process [18], which means various reagents can be entrapped into the microporous network to create a chemical sensor [19-23], and it is directly formed on an electrode surface and thus making it a structured electrode ideally suited for electroanalytical applications.

\section{Experimental}

\subsection{Templated Silica Film Formation}

2.1.1. Spin Cast Method for Colloidal Crystal Formation. Gold electrodes (EMF, Ithaca NY) were cut to $\sim 1.5 \mathrm{~cm} \times 1.5 \mathrm{~cm}$ and then cleaned by successive sonications in ethanol, and water twice for 15 minutes each followed by plasma cleaning at $10 \mathrm{~W}$ (Southbay PE-2000, DC bias of $-100 \mathrm{~V}$ ) to make the slides hydrophilic. The silica sol was prepared by stirring $0.25 \mathrm{~mL}$ tetramethoxysilane (TMOS, 99\%, Acros), $1.20 \mathrm{~mL}$ methanol $(\mathrm{MeOH}), 1.15 \mathrm{~mL}$ water, and $0.015 \mathrm{~mL} 0.1 \mathrm{M}$ $\mathrm{HCl}$ for 30 minutes and then aged for two days. Sodium dodecylsulfate (SDS) was added $(5 \mathrm{mM})$ to the sol before it was mixed with $0.4 \mu \mathrm{m}$ polystyrene latex spheres (PS, from Invitrogen, formally Interfacial Dynamics Company (IDC), sulfate stabilized, $8 \%)$ at a $1: 1(\mathrm{v} / \mathrm{v} \%)$ ratio. The SDS was added as a wetting agent to improve the quality of the sphere packing [7]. The solution was viciously mixed, then pipetted on to the surface of a clean gold electrode, and spin coated at $3000 \mathrm{rpm}$ for 30 seconds. After the films were dried overnight on the bench top, they were submerged in chloroform for three hours to remove the PS spheres.

\subsubsection{Evaporative Method for Colloidal Crystal Formation.} Gold $(\mathrm{Au})$ electrodes were cut into $\sim 1.5 \mathrm{~cm} \times 1.5 \mathrm{~cm}$ squares and cleaned by successive sonications in 2-propanol and water followed by boiling piranha solution $(7: 3)$ for 5 minutes. (Caution: piranha solutions are extremely dangerous and react violently with organic materials). The electrodes were rinsed well with water and ethanol and soaked in a $5 \mathrm{mM}$ cysteamine in ethanol (EtOH) for 24 hours. To form the colloidal crystal, a modified version of the vertical deposition method was used [24]. In this work, the modified electrode was vertically placed in a glass vial, and a mixture of ethanol and $0.5 \mu \mathrm{m}$ spheres in a $33: 1 \mathrm{~V} / \mathrm{V}$ ratio was added. The solution was slowly evaporated at $50^{\circ} \mathrm{C}$ by placing the vial in a thermostated oven or a water bath. A closed water bath was preferred because it tended to minimize fluctuations in temperature and ambient humidity. Evaporation took approximately 24 hours and resulted in a well-packed, single layer (or near single layer) of spheres on the gold electrode.

To form the silica nanowells on the surface of the gold electrode, a silica sol $(0.2 \mathrm{~mL}$ TMOS, $0.06 \mathrm{~mL}$ MTMOS 


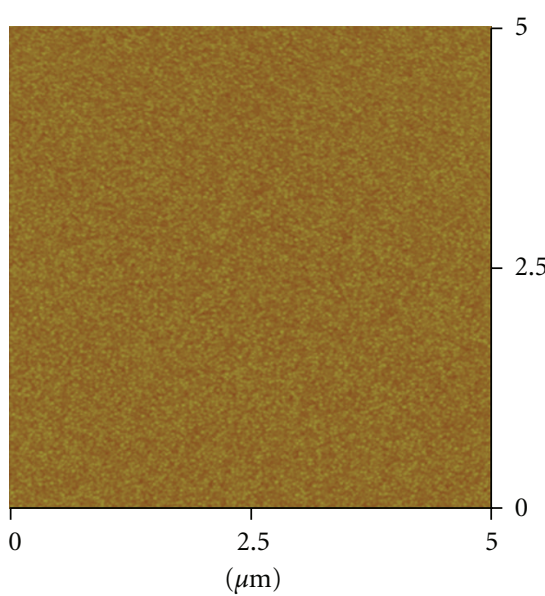

(a)

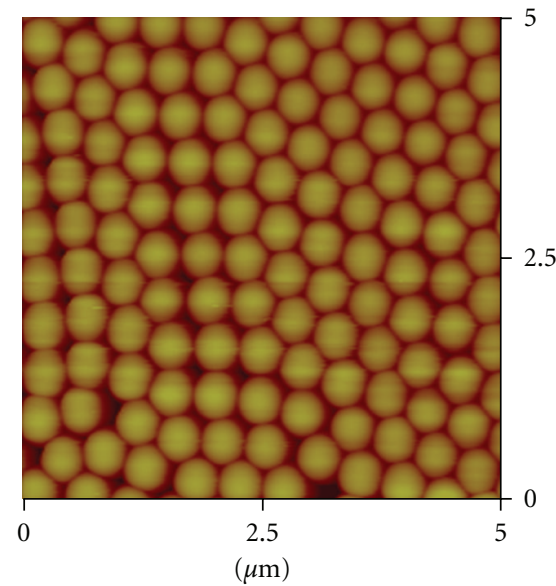

(b)

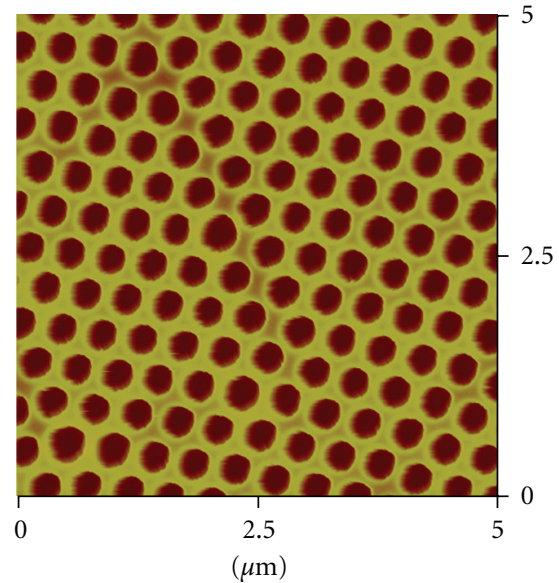

(c)

Figure 2: Tapping mode AFM images ( $5 \mu \mathrm{m} \times 5 \mu \mathrm{m}$ ) of (a) a bare gold electrode, (b) hexagonally packed PS spheres in a silica film-coated gold electrode, and (c) the nanowells formed after chemical removal of the PS spheres.

(methyltrimethoxysilane, 97\%), $0.1 \mathrm{~mL} \quad \mathrm{MeOH}, \quad 0.3 \mathrm{~mL}$ water, and $0.06 \mathrm{~mL} 0.1 \mathrm{M} \mathrm{HCl}$, stirred for 1 hour) was diluted with ethanol $1: 100$, and the diluted sol was then spin coated over the packed spheres at 8000 or $6000 \mathrm{rpm}$ for 60 seconds. To ensure the sol effectively penetrated the voids in the colloidal crystal, the substrates were first plasma cleaned for 2 minutes at $10 \mathrm{~W}(\mathrm{DC}$ bias of $-100 \mathrm{~V})$, and the diluted sol also was allowed to rest on the substrate for one minute prior to electrode rotation. The films were dried overnight, and the PS spheres were removed via soaking in chloroform to form the silica nanowells on the gold electrode. In some cases, to further hydrolyze the sol, $10 \mu \mathrm{L}$ of $0.16 \mathrm{M} \mathrm{KOH}$ was added to the sol and stirred for an additional 30 minutes prior to spin coating.

2.2. Electroless Gold Deposition. To insure that all PS was removed from the film, the substrates were rinsed with fresh chloroform and plasma cleaned for 2 minutes at $15 \mathrm{~W}$ (DC bias of $-150 \mathrm{~V}$ ) prior to submerging in a $1: 100$ $(\mathrm{v} / \mathrm{v} \%)$ solution of hydrogen tetrachloroaurate $(1 \mathrm{mM})$ and $\mathrm{NH}_{2} \mathrm{OH} \cdot \mathrm{HCl}(0.45 \mathrm{mM})$ for varying time lengths. During deposition, the solution was placed on a shaker at $180 \mathrm{rpm}$. The solution was replaced with a fresh solution at 15 minute intervals. The films were rinsed with water then dried on the bench before further characterization.

2.3. Electrochemistry. Electrochemistry was performed in a three-electrode cell using a saturated $\mathrm{Ag} / \mathrm{AgCl}$ electrode as a reference and a Pt wire as the auxiliary on a $\mathrm{CH}$ instruments potentiostat. The electrode area was defined by a quarter inch diameter hole punched into a piece of Teflon tape and copper tape was attached to the edge of the gold slide as a lead. Ferrocene methanol $(1 \mathrm{mM})$ and potassium ferricyanide $(1 \mathrm{mM})$ in $0.1 \mathrm{M} \mathrm{KCl}$ were used as the redox probes.

\section{Results and Discussion}

3.1. Overview. Previous work in our lab has shown that silica nanowells can be formed on glassy carbon electrodes by templating with latex spheres $[8,9]$. Because these nanowells are open at the bottom, thus exposing the electrode underneath, they can be used as nanosized reaction vessels to do electrochemistry [8]. In essence, they are the nanosized equivalent of a round-bottom flask containing an immobilized microelectrode as depicted in the cartoon shown in Figure 1. In the present work, two methods were used to form the silica nanowells. Both methods utilize the sol-gel process [18], which is a versatile method used to prepare inorganic and organic-inorganic hybrid materials [19-23]. The first method (Figure 1, method 1) is the more traditional way to make sol-gel-derived films, which first involves doping the silica sol with latex spheres and spin coating the composite sol on the electrode surface. In the second method (Figure 1, method 2), a near monolayer of spheres is formed first, followed by spin coating an undoped silica sol on this surface. These structured electrodes are then used as reaction vessels to electrolessly grow gold nanoparticles of varying height and width, defined in part by the size of the nanowell and the electroless growth conditions. It is known in the literature that exposure of gold nanoparticles to $\mathrm{HAuCl}_{4}$ in the presence of the reducing agent $\mathrm{NH}_{2} \mathrm{OH}$, either in solution or immobilized on a substrate, results in particle growth [25]. It was hypothesized that the exposed gold electrode located at the bottom of the silica nanowell can be used as a reduction site for gold deposition, and nanoparticles were not necessary to achieve this affect. This same method could be applied to any gold surface of limited dimensions such as the small area of gold exposed at the bottom of the nanowell upon template removal. The size/microstructure of the gold deposits can be controlled by the length of exposure time to the growth 


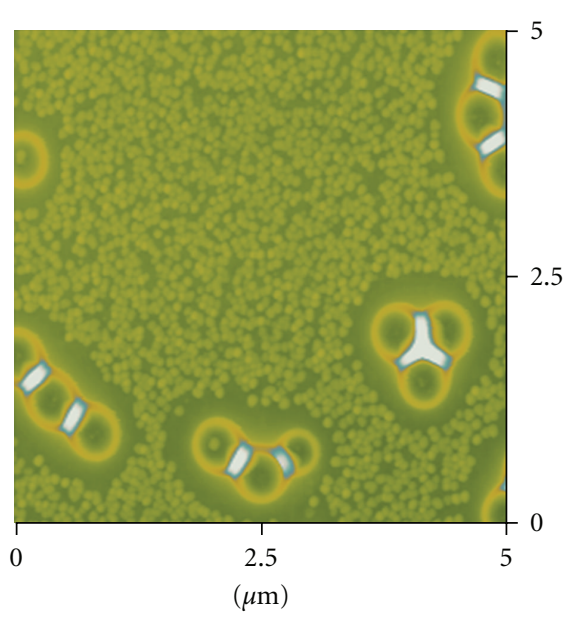

(a)

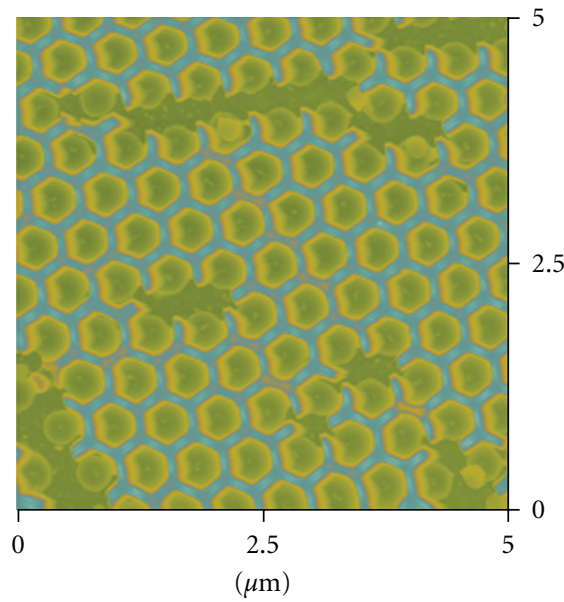

(b)

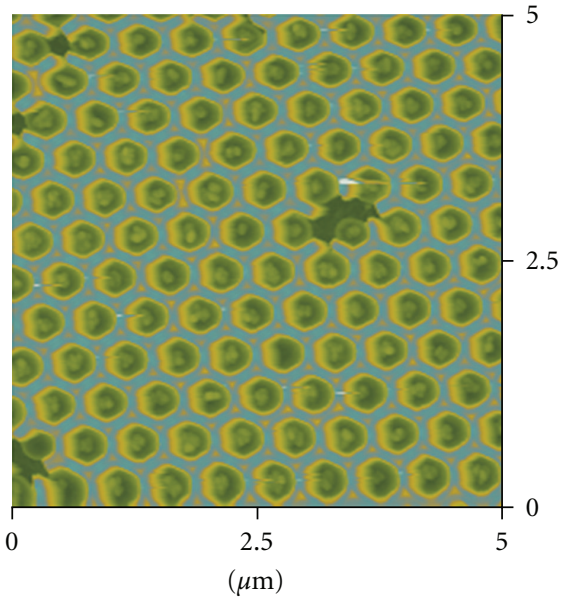

(c)

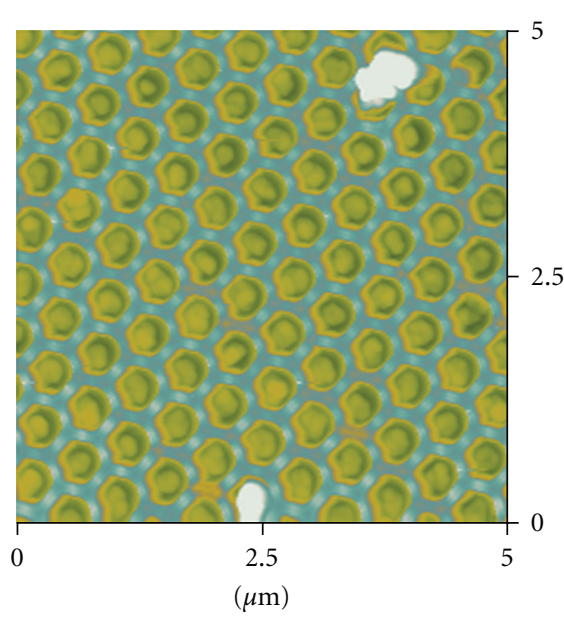

(d)

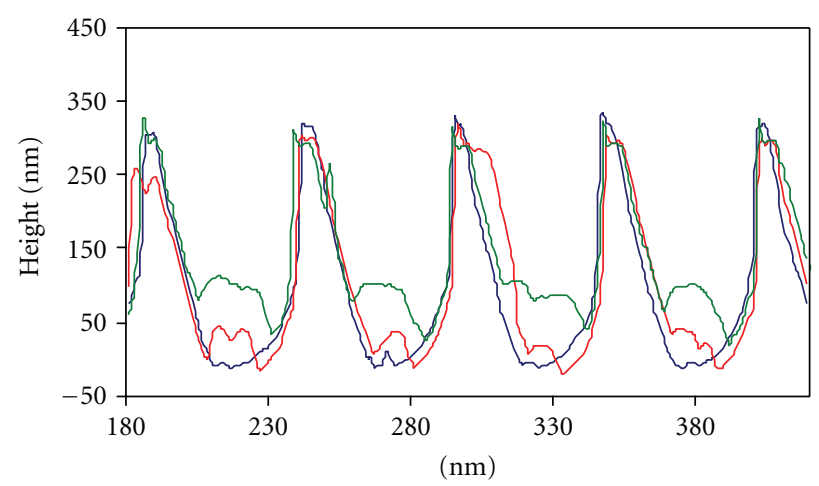

(e)

FIGURE 3: $5 \mu \mathrm{m} \times 5 \mu \mathrm{m}$ AFM images of gold deposited on (a) a bare gold electrode (electroless deposition for 6 minutes) and in silica nanowells grown by electroless deposition for (b) 1 minute, (c) 15 minutes, and (d) 3-hour exposure times. (e) Cross-section plots overlaid for clarity (blue $=1 \mathrm{~min}$, red $=15 \mathrm{~min}$, and green $=3$ hours ).

solution (Figure 1, bottom) and the area exposed to solution upon removal of the PS latex sphere. No prior modification of the surface is necessary.

\subsection{Gold Nanoparticles Formed in Silica Nanowell Electrodes} Prepared via Method 1. In this method, silica sols are doped with PS spheres and then spin cast on the electrode surface. The packing of the spheres on the surface of the electrode depends on many factors including surface charge (electrostatic repulsion), surface hydrophilicity, and surface cleanliness $[6,7]$. In this work, gold electrodes and sulfatestabilized PS spheres are used. Figure 2(a) shows a tapping mode atomic force microscope (AFM, Veeco Multimode SPM) image of the surface of a bare gold electrode. As can be seen, the bare electrode consists of a flat surface with small, random roughness. After spin coating, the silica sol/latex sphere mixture on the gold electrode, localized regions of the surface shows a hexagonally packed monolayer of the PS spheres, Figure 2(b). After template removal, silica nanowells are formed on the surface as shown in Figure 2(c). The depth of the nanowell largely depends on the speed at which the sphere-doped sol is cast, the viscosity of the sol, and size of the latex sphere [8]. At a spin speed of $3000 \mathrm{rpm}$ and a sphere size of $400 \mu \mathrm{m}$, the nanowells have an average dimension of $500 \mathrm{~nm}$ in diameter and a well depth of $300 \mathrm{~nm}$. These nanowells are open at the top (evident in the AFM images) and the bottom (evident from AFM section scans and gold growth (described below)), thus exposing the underlying nanostructured surface.

The nanowells, such as those shown in Figure 2(c) can be used as reaction vessels to electrolessly grow gold nanoparticles. In prior work, we utilized a similar electroless deposition procedure to grow nanostructured gold wires in micron size channels formed from bacteria [26]. Figure 3(a) shows an area of the substrate not covered by packed spheres, which, due to the generally poor interaction between gold and silica, results in almost no film formation [27]. Exposure of the bare gold electrode surface to the gold growth solution 


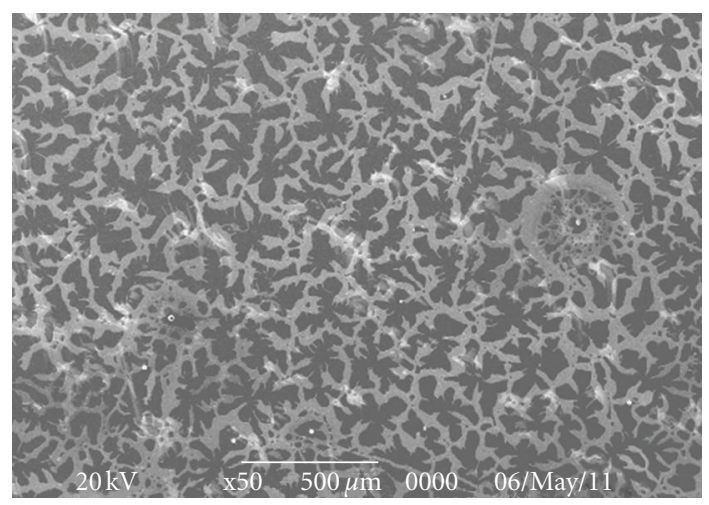

(a)

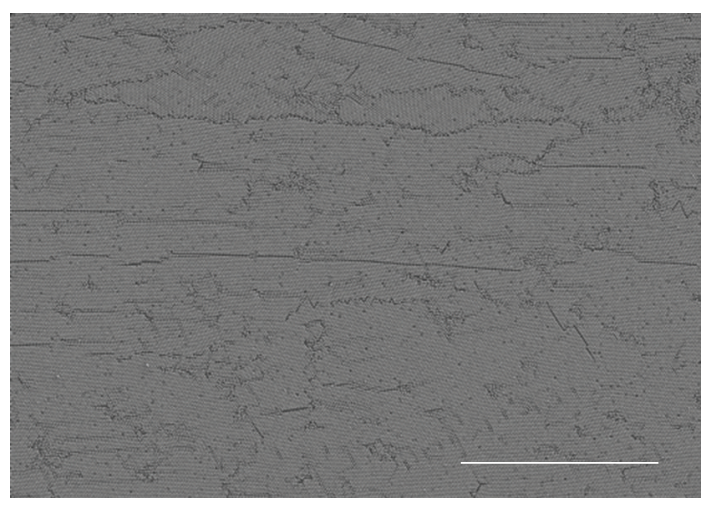

(b)

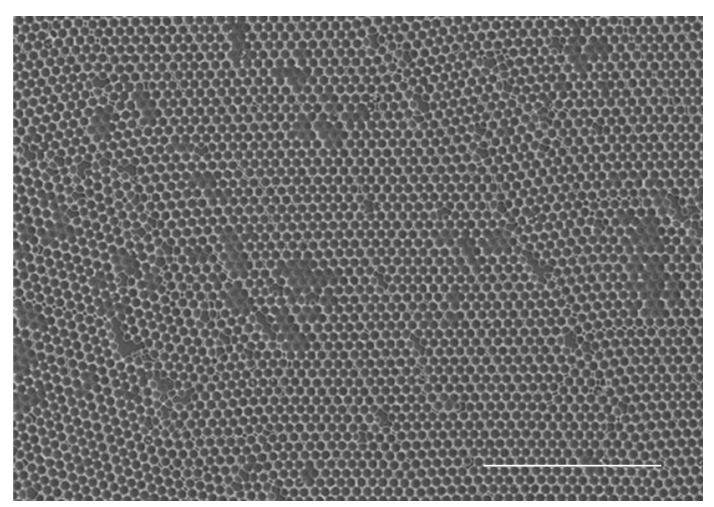

(c)

FIGURE 4: SEM images of typical sphere packing on a gold electrode using (a) the spin-coating method and (b) the vertical deposition method. (c) SEM of the nanowells formed in a film prepared via the vertical deposition method. (Scale bar: (a) $500 \mu \mathrm{m}$, (b) $50 \mu \mathrm{m}$, and (c) $10 \mu \mathrm{m})$.

resulted in increased roughness of the surface, with the occasional large asymmetrical gold deposit. The electroless deposition of gold within the nanowells, however, was found to be markedly different compared to that observed at a bare gold electrode, as in Figures 3(b) and 3(c). Electroless deposition in the nanowells produced (near) sphere-like deposits of consistent size throughout the film. The growth of single particles in the nanowells is likely due to there being only a few exposed points of roughness from the underlying substrate. A single small growth is formed at those points, and then the particle increases in the same manner as an anchored, pregenerated nanoparticle formed in solution, free from competition and eventual merger with adjacent deposition sites as seen in the bare substrate [28]. This growth progression is seen in the time-dependent growth study. A one-minute exposure results in the formation of a deposit with an observable height of about $\sim 20 \mathrm{~nm}$ (Figure 3(b)), which is similar to gold nanoparticles typically generated in solution [29]. As the exposure time continues, the particle increases in size while retaining its basic shape (Figure 3(c)). There appeared to be a limit to this growth, however, as it was observed that growth would not continue past a height of $\sim 150 \mathrm{~nm}$ (Figure 3(d)), even when left in the growth solution overnight (data not shown). Figure 3(e) shows an overlay of the cross-sections of the nanowells before and after electroless deposition, ranging from particle formation to the growth limit. As can be seen, the conductive domain located at the bottom of the nanowell progressively increases as the incubation time in the growth solution increases.

3.3. Gold Nanoparticles Formed in Silica Nanowell Electrodes Prepared via Method 2. The spin-coating method, while simple and easy to do, does not produce nanowell arrays with significant long-range order on gold electrodes that would likely be required for electroanalytical applications. Part of the problem likely results from the surface of gold not being easily wetted by the silica sol, as well as the lack of available hydroxyl groups for the silica to bind with [27, 30]. To address this shortcoming, the method used to pack the colloidal crystal on the electrode surface was separated from silica film formation. There have been many methods described in the literature for the formation of colloidal crystals on gold with long-range order $[2,6,7,31]$. Many of these published methods are ideally suited for forming multilayer films, which would be more challenging in this study. In this work, the method with the greatest success for forming a monolayer or near-monolayer of PS spheres with longrange order is a variant of the vertical deposition/solvent 


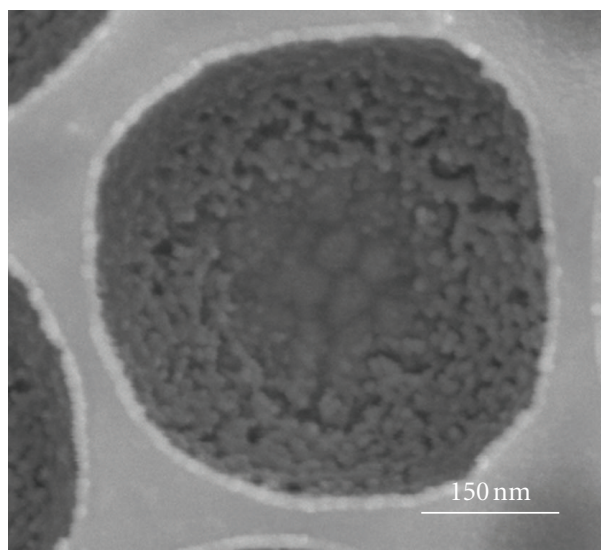

(a)

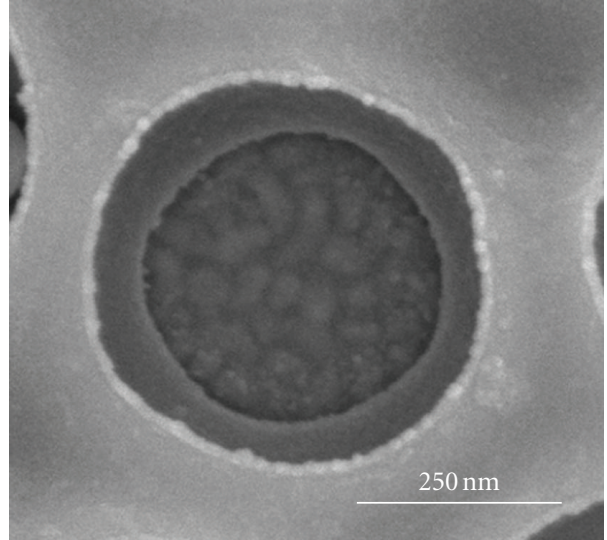

(b)

Figure 5: SEM images of (a) a narrow and (b) a wide conductive domain located at the bottom of the silica nanowells on a gold electrode. (Scale bar: (a) $150 \mathrm{~nm}$, (b) $250 \mathrm{~nm}$ ).

evaporation method $[2,24]$. In this method, the substrate is vertically positioned in a vial containing a dilute suspension of spheres in ethanol $[2,24]$. As the solvent slowly evaporates, a compact layer of spheres remain on the surface [24]. In this work, the concentration of latex spheres in ethanol was kept relatively low (1:33 V/V PS spheres: EtOH), and evaporation took place at an elevated temperature $\left(50^{\circ} \mathrm{C}\right)$. To insure a steady evaporation rate, the evaporation setup was heated in a closed water bath to provide consistent heating and humidity. In contrast to a room-temperature-based deposition, a packed monolayer (or near monolayer) of PS spheres can be formed in 24 hours. This method produced a vastly superior colloidal crystal with long-range packing of spheres, mostly in a single layer, compared to the spincoating method. Figure 4 shows representative $157 \times 110 \mu \mathrm{m}$ scanning electron microscope images (SEM, Hitachi FE-SEM SU-70, $5 \mathrm{kV}$, platinum/gold sputter to minimize charging effects) of a single (or near single) layer of PS spheres on a gold electrode formed by spin coating the sphere-doped sol on a gold electrode (Figure $4(\mathrm{a})$ ) or via the vertical deposition method (Figure 4(b)). While the spin-coated packed electrode shows good packing in localized areas, the packing is weblike over the entire surface, leaving a large amount of the substrate exposed. The vertical deposition method, however, exhibits good packing on both the microscopic and macroscopic length scales (Figure 4(b)). In Figure 4(c), an SEM of the nanowells formed after template removal is shown.

Once the colloidal crystal was formed on the gold electrode, a silica sol could be spin cast directly over this layer of spheres to ultimately form the silica nanowells on the gold electrode (Figure 4(c)) [26]. The silica nanowells produced by this method have many similarities to that of the doping method (described above) in that dimensions of the nanowells are similar. One major difference is in the variation of the diameter of the underlying exposed electrode surface (the conductive domain), which has consequences for the electroless deposition of gold. The variables that will likely influence the diameter of the bottom of the nanowell include the degree at which the sol is hydrolyzed and condensed (its age), the viscosity of the sol (dilution factor), the time the sol is placed on the surface before the electrode is rotated, and the speed at which the sol is cast over the packed spheres. Fresh silane precursors and faster spin speeds $(8000 \mathrm{rpm})$ tend to produce wider bottoms, while aged silane (one day) and slower speeds (6000 rpm) produce narrower bottoms. Because speed influences film thickness, the original sol was diluted more when the spin speed was decreased to retain nearly the same nanowell depth. Figure 5 shows representative examples of the sizes of the conductive domain formed at the bottom of the nanowells on a gold electrode. The "wide bottom" nanowells expose a substrate area of about $70-80 \%$ that of the mouth of the nanowell, whereas the "narrow bottom" nanowells expose roughly 30$40 \%$. The sides of the nanowell shown in Figure 5(a) exhibit a more particle-like surface relative to that observed in the nanowell shown in Figure 5(b). These observed differences in the microstructure of the silica network are attributed to differences in the aging of the silane precursors prior to making the sol. In Figure 5(b), the silane precursors were freshly removed from a nitrogen environment and immediately used to prepare the sol, whereas in Figure 5(a), the silanes were partially hydrolyzed via exposure to air prior to use.

Films that exhibited large conductive domains at the bottom of the nanowells produced gold deposits with asymmetric shapes and sizes (Figure 6(b) inset). Films with smaller conductive domains produced deposits consistent with those observed with the doped films (Figure 3), with a single sphere-like particle (Figure 6(a) inset). The growth in the wide bottom appears to be a hybrid of the growth patterns on the bare gold surface and in the narrow bottom wells. The shape of the deposit reflects the natural roughness of the gold surface under the film but is able to grow past the point commonly seen on the bare surface, similar to the nanowells with smaller conductive domains. It is believed that this is the result of the wider conductive domains having several initial growth sites within the nanowell that merge 


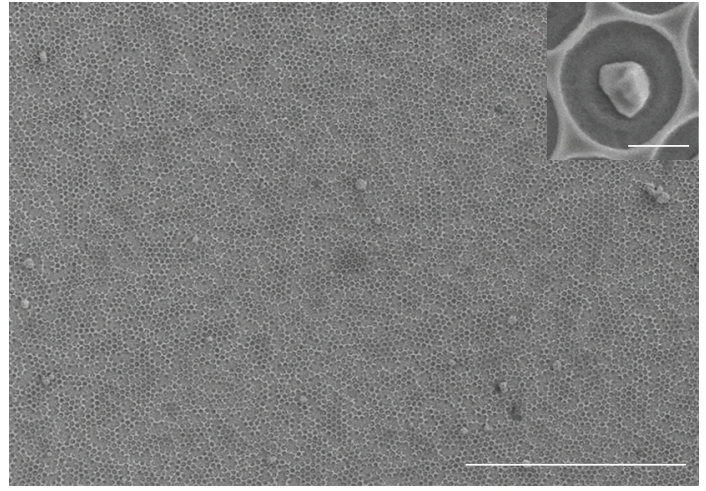

(a)

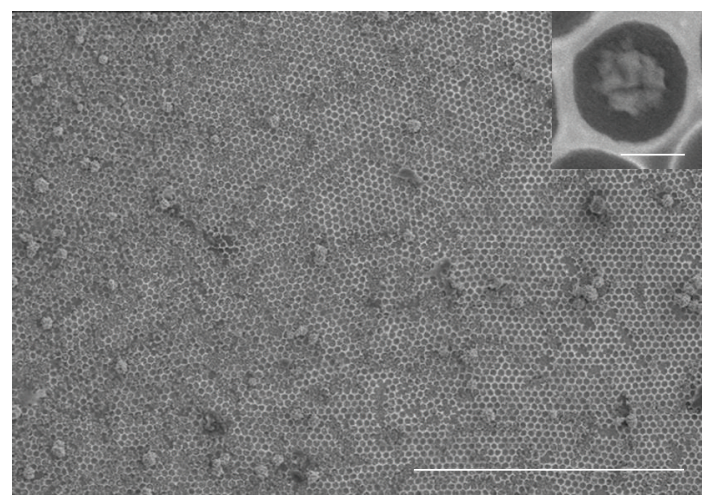

(b)

Figure 6: SEM images of (a) narrow bottom and (b) wide bottom films. Insets display representative gold deposits within silica nanowells. (Scale bars: $20 \mu \mathrm{m}$, inset: $250 \mathrm{~nm}$ ).

together at longer growth times. By combining the vertical deposition method of film formation with the gold growth method utilized in the spin-coated slides, a large, well-packed array of nanowells containing gold particles of controllable size were formed (Figure 6).

3.4. Electrochemistry. The voltammetry of ferrocene methanol $\left(\mathrm{FcCH}_{2} \mathrm{OH}\right)$ and potassium ferricyanide $\left(\mathrm{Fe}(\mathrm{CN})_{6}{ }^{3-}\right)$ were examined at electrodes prepared via method 2. In Figure $7(\mathrm{a})$, cyclic voltammograms (CVs) for $\mathrm{FcCH}_{2} \mathrm{OH}$ at a bare gold electrode and a silica film/sphere-coated electrode can be observed. As expected, at a bare gold electrode, the voltammogram has a diffusion-controlled shape characteristic of a redox probe in solution transferring electrons at the surface of a large planar electrode. At the sphere-coated electrode, different electrochemical responses were observed depending on the quality of the packing of the latex spheres (and the redox probe, described below). When the electrode contained closely spaced defects, such as those formed at surface dislocations and areas not coated with silica, and/or at pinholes between spheres, the $\mathrm{CV}$ of $\mathrm{FcCH}_{2} \mathrm{OH}$ looks almost identical to that obtained at a bare electrode. In this case, the diffusion layers at the individual defect sites overlap and the surface appears as one big electrode $[32,33]$. In contrast, when the electrode

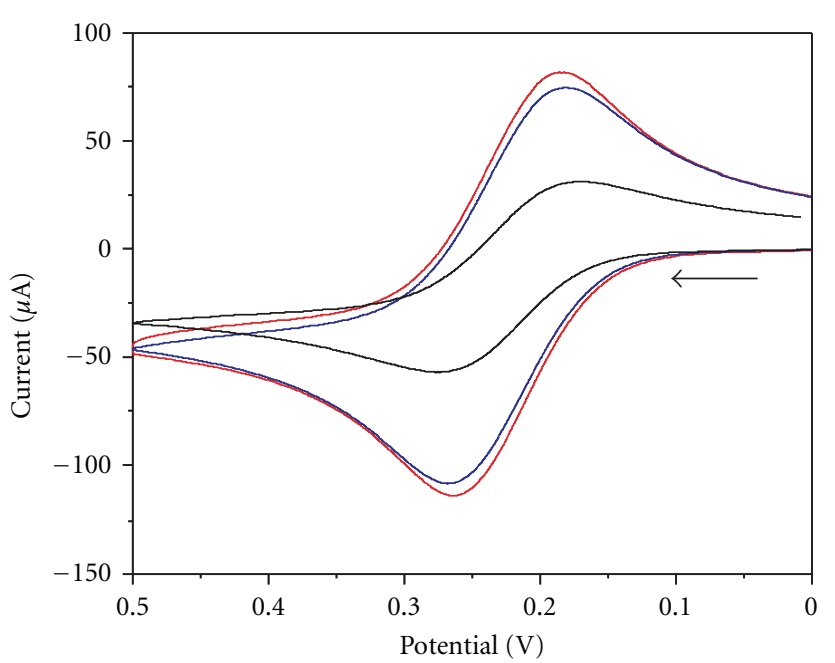

(a)

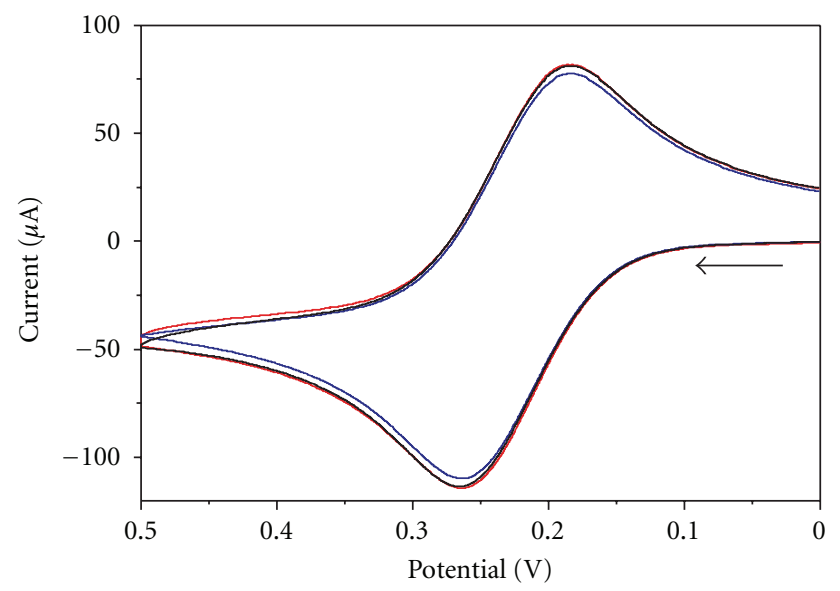

(b)

Figure 7: CVs of ferrocene methanol $(1 \mathrm{mM})$ in $0.1 \mathrm{M} \mathrm{KCl}$ at $0.1 \mathrm{~V} / \mathrm{s}$. (a) Red: bare gold, blue: silica film/sphere-coated electrode with many defects in the colloidal crystal, and black: silica film/sphere-coated electrode with fewer defects in the colloidal crystal. (b) Red: bare gold, blue: after sphere removal via soaking in chloroform for $3 \mathrm{hrs}$, and black: after particle growth for 45 minutes.

is covered with a densely packed layer of spheres with few defects and better long-range packing, the oxidation of ferrocene methanol is hindered, and the voltammetric curve is suppressed relative to that observed at a bare electrode. Upon removal of the spheres leaving behind a closely spaced array of nanowells, the voltammetry once again looks like that observed at a bare electrode because of the overlap of diffusion layers at the closely spaced nanowells, as in Figure 7(b) [8]. Once the spheres are removed, the quality of the packing of the colloidal crystal (i.e., the presence of defects/pin holes) cannot be assessed from the voltammetry because the electrochemistry that takes place at a defect site cannot be distinguished from the electrochemistry that takes place in the nanowell [8]. Further growth of the exposed gold electrode via electroless deposition for $45 \mathrm{~min}$ does not 


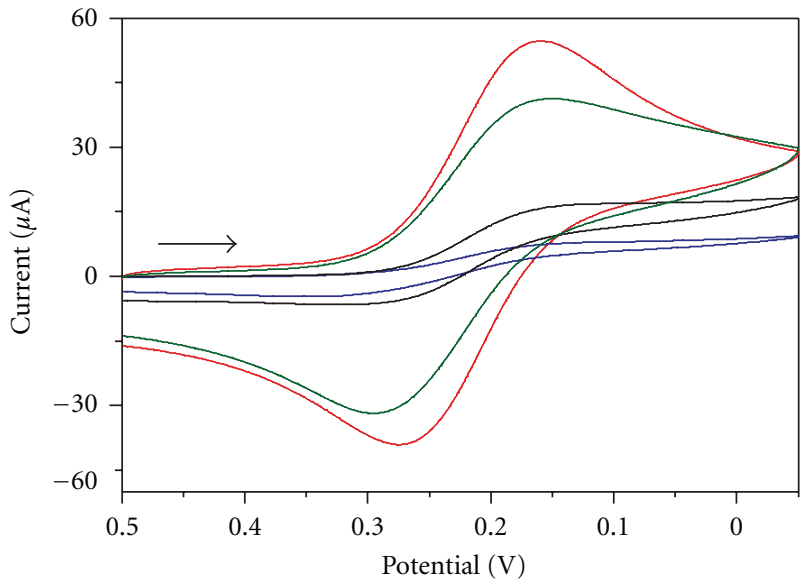

Figure 8: CVs of potassium ferricyanide $(1 \mathrm{mM})$ in $0.1 \mathrm{M} \mathrm{KCl}$ at $0.1 \mathrm{~V} / \mathrm{s}$. Red: bare gold, blue: silica film/sphere-coated electrode, black: silica film after sphere removal via soaking in chloroform for $3 \mathrm{hrs}$, and green: silica film after gold particle growth for 45 minutes.

produce distinct changes: the voltammetry of $\mathrm{FcCH}_{2} \mathrm{OH}$ looks identical to that observed at nanowell electrode.

Very different results were observed using $\mathrm{Fe}(\mathrm{CN})_{6}{ }^{3-}$ as the redox probe. $\mathrm{Fe}(\mathrm{CN})_{6}{ }^{3-}$ is very similar in size to $\mathrm{FcCH}_{2} \mathrm{OH}$, but is negatively charged. Thus, electrostatic interactions will be more important compared to the case for $\mathrm{FcCH}_{2} \mathrm{OH}$, which is initially neutral. Under nearneutral $\mathrm{pH}$ conditions, the silica matrix will be negatively charged. Figure 8 shows the CVs at a bare electrode, a silica film/sphere-coated electrode, the nanowell electrode, and the nanowell electrode with the conducting $\mathrm{Au}$ domains enlarged. In contrast to that observed using $\mathrm{FcCH}_{2} \mathrm{OH}$, a significantly diminished response is observed for $\mathrm{Fe}(\mathrm{CN})_{6}{ }^{3-}$ at the silica/sphere-coated electrode. The voltammetric curve exhibits a sigmoidal-shaped response indicative of the presence of pinholes/defects that are relatively far apart. After removal of the spheres, the voltammetric shape remains sigmoidal-like but increases in size. The negatively charged silica matrix obviously hinders access of $\mathrm{Fe}(\mathrm{CN})_{6}{ }^{3-}$ to the underlying gold electrode relative to that observed for $\mathrm{FcCH}_{2} \mathrm{OH}$. By increasing the size of the Au conducting domain via electroless deposition, electrostatic repulsion between the redox probe and the silica framework is lessened, and the $\mathrm{CV}$ of $\mathrm{Fe}(\mathrm{CN})_{6}{ }^{3-}$ more closely resembles that observed at the bare electrode.

\section{Conclusion}

Nanostructured gold electrodes were created through the selective electroless deposition of gold into nanowells formed in a silica film on a gold electrode. The size and shape of the deposited particles were controlled via exposure time and changes in film characteristics. Method 1, which involved spin coating a doped sol on a gold electrode, was simple and easy and yielded nanowells that all had similar size conductive domains. When used as a chemical reactor to electrolessly expand the conductive domain, uniform gold deposits were obtained. The nanowells, however, were not uniformly distributed/packed across the entire surface of the electrode, potentially limiting their application in electroanalytical chemistry. Method 2, while a more timeconsuming 2-step method, yielded a much more uniformly packed array of nanowells. The conductive domains located at the bottom of the nanowells, however, varied in size. Both nanowells with large conductive domains and nanowells with small conductive domains were obtained by judiciously changing the sol-gel processing conditions. The size of the conductive domain significantly influenced the size and structure of the gold deposit electrolessly formed at the bottom of the well. Initial electrochemistry experiments using two different redox probes, ferrocene methanol and potassium ferricyanide, showed interesting results. Most significantly, the shape of the cyclic voltammetric curve and the magnitude of the faradaic current were strongly dependent on the long-range packing and the presence of defects in the film and the redox probe in solution. For potassium ferricyanide, enlargement in the conductive domains lessens electrostatic repulsion effects observed from the silica network and results in a more traditional-shaped voltammetric similar to that obtained a bare electrode.

\section{Acknowledgments}

The authors gratefully acknowledge support of this work from the National Science Foundation (CHE-0618220, CHE0847613). A. E. Rue thanks Altria for a fellowship. They also acknowledge the support of the VCU Nanomaterials Core Characterization (NCC) facility and Dr. Dmitry Pestov and Ms. Bo Zhao for their help with the SEM.

\section{References}

[1] A. Walcarius, "Template-directed porous electrodes in electroanalysis," Analytical and Bioanalytical Chemistry, vol. 396, no. 1, pp. 261-272, 2010.

[2] A. Walcarius and A. Kuhn, "Ordered porous thin films in electrochemical analysis," TrAC-Trends in Analytical Chemistry, vol. 27, no. 7, pp. 593-603, 2008.

[3] A. Walcarius, "Electroanalytical applications of microporous zeolites and mesoporous (organo)silicas: recent trends," Electroanalysis, vol. 20, no. 7, pp. 711-738, 2008.

[4] M. M. Collinson, "Sol-gel strategies for the preparation of selective materials for chemical analysis," Critical Reviews in Analytical Chemistry, vol. 29, no. 4, pp. 289-311, 1999.

[5] N. K. Raman, M. T. Anderson, and C. J. Brinker, "Template-based approaches to the preparation of amorphous, nanoporous silicas," Chemistry of Materials, vol. 8, no. 8, pp. 1682-1701, 1996.

[6] Y. Li, W. Cai, and G. Duan, "Ordered micro/nanostructured arrays based on the monolayer colloidal crystals," Chemistry of Materials, vol. 20, no. 3, pp. 615-624, 2008.

[7] Y. Xia, B. Gates, Y. Yin, and Y. Lu, "Monodispersed colloidal spheres: old materials with new applications," Advanced Materials, vol. 12, no. 10, pp. 693-713, 2000.

[8] M. Kanungo, P. N. Deepa, and M. M. Collinson, "Templatedirected formation of hemispherical cavities of varying depth and diameter in a silicate matrix prepared by the sol-gel 
process," Chemistry of Materials, vol. 16, no. 25, pp. 5535$5541,2004$.

[9] A. N. Khramov and M. M. Collinson, "Sol-gel preparation of macroporous silica films by templating with polystyrene microspheres," Chemical Communications, no. 8, pp. 767-768, 2001.

[10] J. R. Li, K. L. Lusker, J. J. Yu, and J. C. Garno, “Engineering the spatial selectivity of surfaces at the nanoscale using particle lithography combined with vapor deposition of organosilanes," ACS Nano, vol. 3, no. 7, pp. 2023-2035, 2009.

[11] W. Ahn and D. K. Roper, "Periodic nanotemplating by selective deposition of electroless gold island films on particlelithographed dimethyldichlorosilane layers," ACS Nano, vol. 4, no. 7, pp. 4181-4189, 2010.

[12] E. Katz, I. Willner, and J. Wang, "Electroanalytical and bioelectroanalytical systems based on metal and semiconductor nanoparticles," Electroanalysis, vol. 16, no. 1-2, pp. 19-44, 2004.

[13] C. M. Welch and R. G. Compton, "The use of nanoparticles in electroanalysis: a review," Analytical and Bioanalytical Chemistry, vol. 384, no. 3, pp. 601-619, 2006.

[14] X. Luo, A. Morrin, A. J. Killard, and M. R. Smyth, "Application of nanoparticles in electrochemical sensors and biosensors," Electroanalysis, vol. 18, no. 4, pp. 319-326, 2006.

[15] J. M. Pingarron, P. Yanez-Sedeno, and A. Gonzalez-Cortes, "Gold nanoparticle-based electrochemical biosensors," Electrochimica Acta, vol. 53, no. 19, pp. 5848-5866, 2008.

[16] J. R. Li and J. C. Garno, "Elucidating the role of surface hydrolysis in preparing organosilane nanostructures via particle lithography," Nano Letters, vol. 8, no. 7, pp. 1916-1922, 2008.

[17] J. R. Li and J. C. Garno, "Nanostructures of octadecyltrisiloxane self-assembled monolayers produced on $\mathrm{Au}(111)$ using particle lithography," Acs Applied Materials \& Interfaces, vol. 1, no. 4, pp. 969-976, 2009.

[18] J. Brinker and G. Scherer, Sol-Gel Science, Academic Press, New York, NY, USA, 1990.

[19] D. Avnir, "Organic chemistry within ceramic matrices: doped sol-gel materials," Accounts of Chemical Research, vol. 28, no. 8, pp. 328-334, 1995.

[20] D. Avnir, S. Braun, O. Lev, and M. Ottolenghi, "Enzymes and other proteins entrapped in sol-gel materials," Chemistry of Materials, vol. 6, no. 10, pp. 1605-1614, 1994.

[21] O. Lev, M. Tsionsky, L. Rabinovich et al., "Organically modified sol-gel sensors," Analytical Chemistry, vol. 67, no. 1, pp. 22A-30A, 1995.

[22] A. Walcarius and M. M. Collinson, "Analytical chemistry with silica sol-gels: traditional routes to new materials for chemical analysis," Annual Review of Analytical Chemistry, vol. 2, pp. 121-143, 2009.

[23] A. Walcarius, D. Mandler, J. A. Cox, M. Collinson, and O. Lev, "Exciting new directions in the intersection of functionalized sol-gel materials with electrochemistry," Journal of Materials Chemistry, vol. 15, no. 35-36, pp. 3663-3689, 2005.

[24] P. Jiang, J. F. Bertone, K. S. Hwang, and V. L. Colvin, "Single-crystal colloidal multilayers of controlled thickness," Chemistry of Materials, vol. 11, no. 8, pp. 2132-2140, 1999.

[25] K. R. Brown and M. J. Natan, "Hydroxylamine seeding of colloidal Au nanoparticles in solution and on surfaces," Langmuir, vol. 14, no. 4, pp. 726-728, 1998.

[26] Z. X. Lu, L. F. Wood, D. E. Ohman, and M. M. Collinson, "Bio-inspired chemical reactors for growing aligned gold nanoparticle-like wires," Chemical Communications, no. 28, pp. 4200-4202, 2009.
[27] J. W. Robertson, M. Cai, and J. E. Pemberton, "Insulating ultrathin silica films formed by a room-temperature sol-gel process," Advanced Materials, vol. 13, no. 9, pp. 662-667, 2001.

[28] K. R. Brown, L. A. Lyon, A. P. Fox, B. D. Reiss, and M. J. Natan, "Hydroxylamine seeding of colloidal Au nanoparticles. 3. Controlled formation of conductive Au films," Chemistry of Materials, vol. 12, no. 2, pp. 314-323, 2000.

[29] K. C. Grabar, R. G. Freeman, M. B. Hommer, and M. J. Natan, "Preparation and characterization of Au colloid monolayers," Analytical Chemistry, vol. 67, no. 4, pp. 735-743, 1995.

[30] S. Sayen and A. Walcarius, "Electro-assisted generation of functionalized silica films on gold," Electrochemistry Communications, vol. 5, no. 4, pp. 341-348, 2003.

[31] P. N. Bartlett, J. J. Baumberg, P. R. Birkin, M. A. Ghanem, and M. C. Netti, "Highly ordered macroporous gold and platinum films formed by electrochemical deposition through templates assembled from submicron diameter monodisperse polystyrene spheres," Chemistry of Materials, vol. 14, no. 5, pp. 2199-2208, 2002.

[32] H. Aluri and M. M. Collinson, "The stability of nonporous and macroporous titania thin films in aqueous electrolyte solutions," Journal of Electroanalytical Chemistry, vol. 651, no. 2, pp. 143-149, 2011.

[33] M. M. Collinson, H. Wang, R. Makote, and A. Khramov, "The effects of drying time and relative humidity on the stability of sol-gel derived silicate films in solution," Journal of Electroanalytical Chemistry, vol. 519, no. 1-2, pp. 65-71, 2002. 


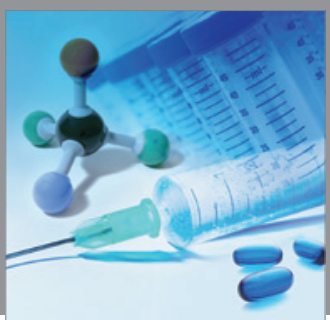

International Journal of

Medicinal Chemistry

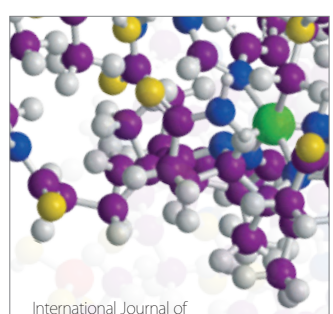

Carbohydrate Chemistry

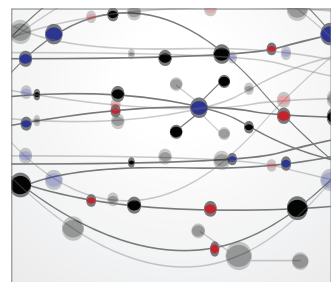

The Scientific World Journal
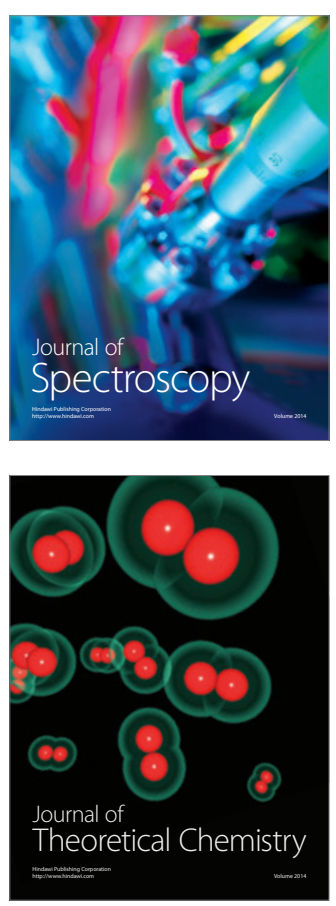
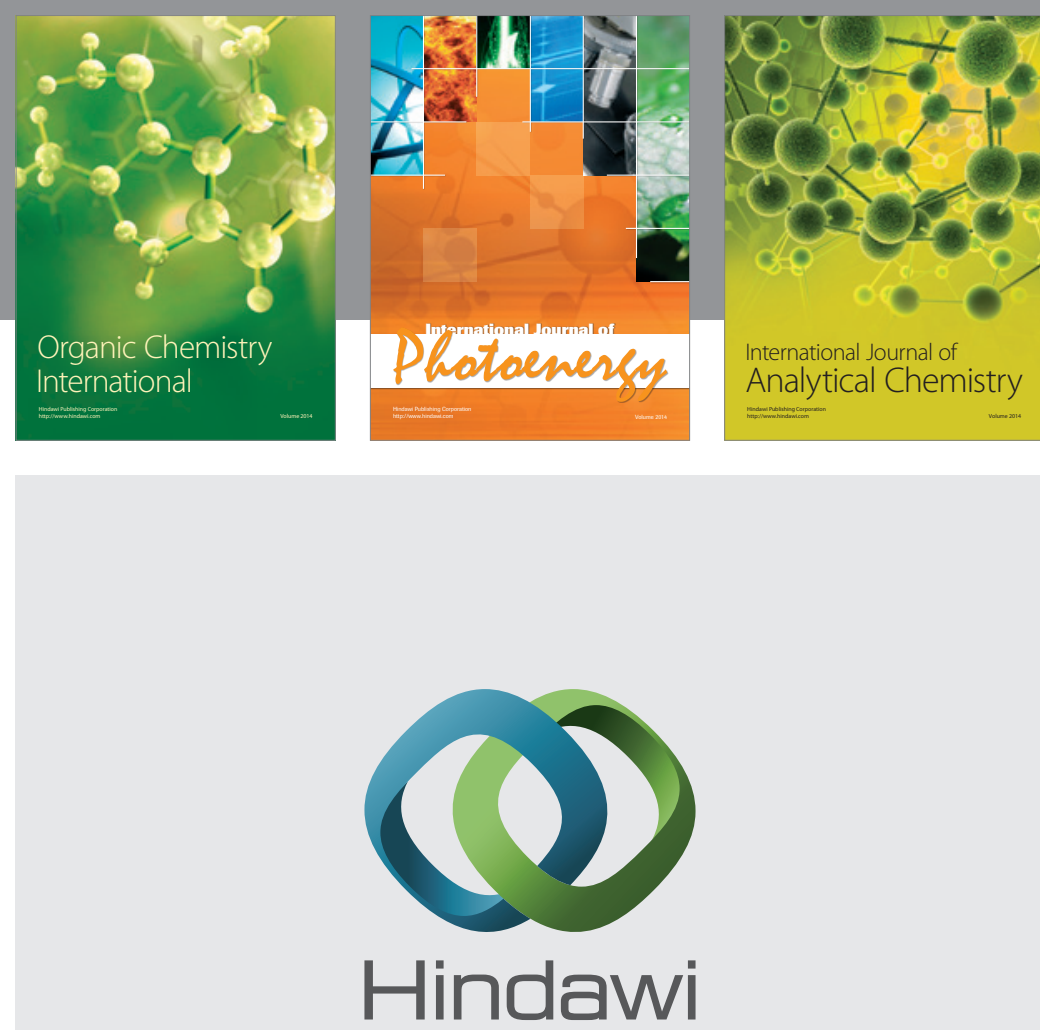

Submit your manuscripts at

http://www.hindawi.com
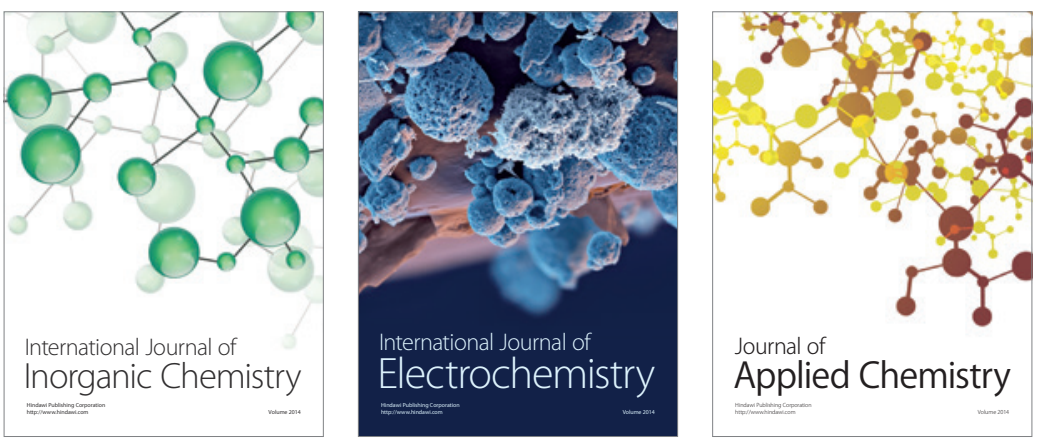

Journal of

Applied Chemistry
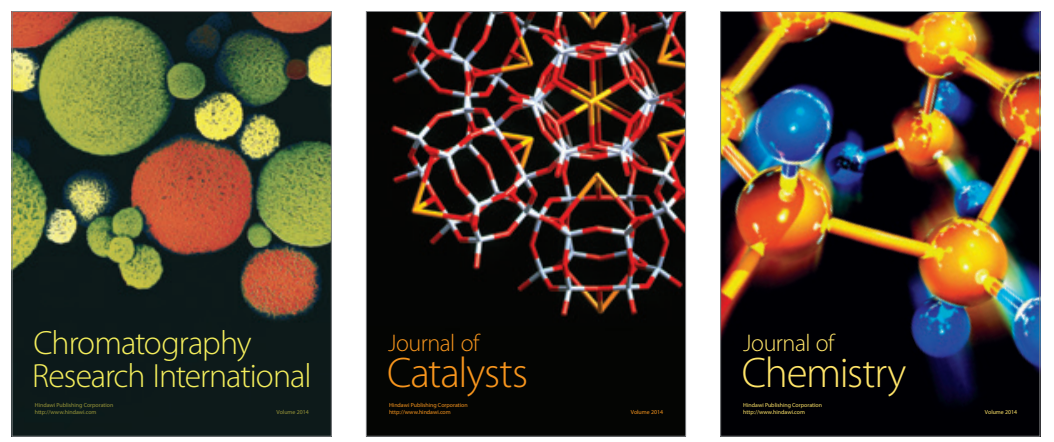
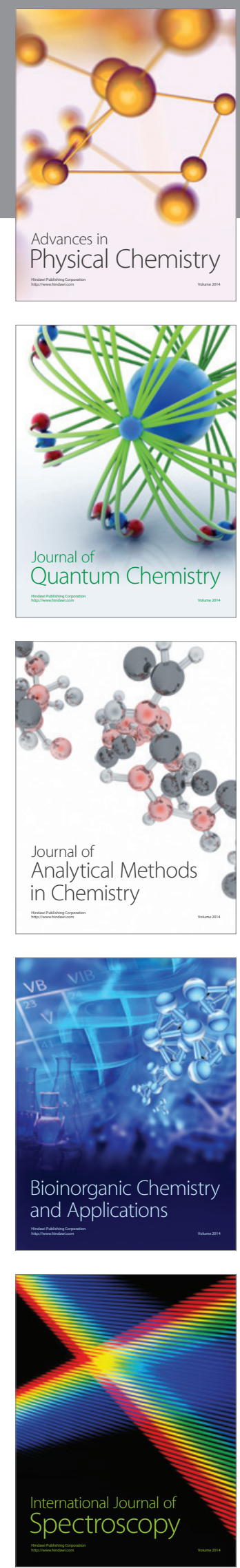